\title{
Cooling Performance of Heat Sinks Used in Electronic Devices
}

\author{
Ibrahim Mjallal, Hussein Farhat, Mohammad Hammoud*, Samer Ali, Ali AL Shaer and Ali Assi \\ Mechanical an Electrical Departments, School of Engineering, Lebanese International University \\ Beirut, Lebanon \\ ${ }^{*}$ Correspondence: mohamad.hammoud@liu.edu.lb
}

\begin{abstract}
Existing passive cooling solutions limit the short-term thermal output of systems, thereby either limiting instantaneous performance or requiring active cooling solutions. As the temperature of the electronic devices increases, their failure rate increases. That's why electrical devices should be cooled. Conventional electronic cooling systems usually consist of a metal heat sink coupled to a fan. This paper compares the heat distribution on a heat sink relative to different heat fluxes produced by electronic chips. The benefit of adding a fan is also investigated when high levels of heat generation are expected.
\end{abstract}

Keywords: Heat sink; Thermal management; Electronic devices

\section{INTRODUCTION}

Electronic equipment have made their way into practically every aspect of modern life, from toys and appliances to highpower computers. Recent increase in processing demands has forced manufacturers to increase the performance and functionality of integrated circuit chips in addition to minimizing their size leading to high power dissipation through smaller packages. This fact made thermal management of integrated circuit chips a critical aspect of successful processor system design, and finding new cooling techniques much more valuable. Moreover, the failure rate of electronic equipments increases exponentially with temperature. Higher temperatures, increases power leakage and degrades the chip reliability. Generally, the global maximum allowable temperatures of various chips range between $85^{\circ} \mathrm{C}$ and $120{ }^{\circ} \mathrm{C}$ [1]. To avoid such problem, packages of integrated circuits must be designed to remove any predicted excessive heat.

Since high temperature reduces performance and degrades chip reliability, the effective management of processor's temperature is an important issue that needs to be investigated. In order to avoid excessive levels of heat and reduce temperature, active and passive cooling techniques are used. In many applications, standard cooling methods could be insufficient for the heat load produced during continuous operation over long periods due to some limitations such as limited thermal conductivity of air for convection and copper for conduction and small spaces decreasing the capabilities performance.

In the present work, thermal management of the chip's temperature is performed using an aluminum heat sink that can dissipate the heat generated by the chip and release it to the surrounding.

\section{Classic CoOling TeChNiques}

For years, various active and passive cooling methods have been applied to cool electronic chips. Active methods are those who need power to work such as forced ventilation using electric fans, liquid cooling through microchannels [2] and heat pipes in fanless systems [3]. While in the Passive cooling methods no power is needed to initiate the cooling process as well as no mechanical moving parts are used. Heat sinks is an attractive option for low power systems. However, size constraints make the use of heat sinks difficult in embedded or mobile systems.

\section{A. Heat Sink}

A heat sink is a passive heat exchanger that transfers the heat generated by an electronic device (chip) to a lower temperature fluid medium [4]. The main role of the heat sink is to maximizes the surface area of contact between the processor and the surrounding cooling medium, by the use of fins. Heat generated by the chip is transferred to the heat sink by conduction, Subsequently this heat is transferred to the surrounding, which is usually air, by convection and radiation. Most often, heat spreaders are used between the chip and the heat sink due to the difference in size between the bottom of the sink and the top of the chip, to ensure more heat dissipation. A heat spreader is a heat exchanger that moves heat between a heat source and another secondary heat exchanger whose surface area and geometry are more favorable than the source. Such a spreader is most often simply a plate made of copper, which has a high thermal conductivity.

Heat sinks as shown in Figure 1 are usually made of metals such as aluminum or copper both having high thermal conductivity to minimize the temperature difference between the chip and the fin's tip. 
The cooling package is modeled as a thermal resistance circuit on basis of $\mathrm{R}$ (thermal resistance) or $\mathrm{C}$ (thermal conductance) where the temperature across two locations can be measured, leading to the calculation of heat dissipation rate [5].

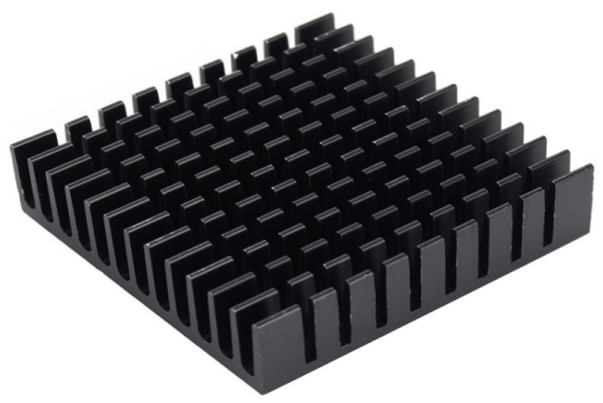

Fig.1. Heat Sink [6]

\section{B. Other Cooling Methods}

Various active and passive cooling methods have been applied to cool electronic chips. Such as forced ventilation, heat pipes, liquid cooled cold plates, immersion cooling, and the use of Phase Change Materials [7].

\section{EXPEREMENTAL SETUP}

The experimental setup is shown schematically in Fig. 2. The heat sink is placed on top of an electronic chip. The dimensions of the sink are $20 \times 20 \times 6 \mathrm{~mm}$. Both the chip and the heat sink are drawn using SolidWorks in order to be used in further simulation.
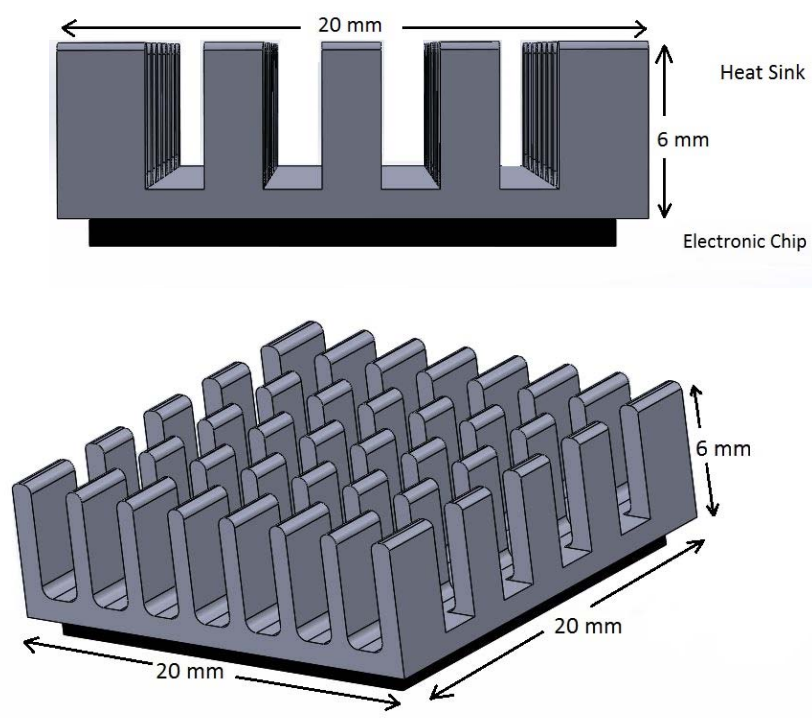

Fig. 2. SolidWorks drawing of the simulated domain.

\section{A. Heat Transfer}

The heat generated by the electronic chip is transferred through the heat sink by conduction and then the heat is carried out by the surrounding air by convection (natural convection is considered in our study) and radiation. Usually, a heat spreader is used to ensure the spreading of the heat all over the whole base of the sink and to overcome the size difference between the electronic chip and the heat sink.

\section{B. Equations}

1) Conduction: is the transfer of energy from the more energetic, to less energetic particles of the same substance or between two substances in contact due to interaction between particles. [6]

$$
q^{\prime \prime}=-K \cdot \nabla T
$$

Where;

$q^{\prime \prime}$ is the heat transfer rate $\left(\mathrm{W} / \mathrm{m}^{2}\right)$

$\mathrm{K}$ is the thermal conductivity $\left(\mathrm{W} / \mathrm{m} .{ }^{\circ} \mathrm{K}\right)$

$$
\nabla T=\left(i \frac{\partial T}{\partial x}+j \frac{\partial T}{\partial y}+k \frac{\partial T}{\partial z}\right)
$$

2) Convection: occurs between a fluid in motion and a bounding surface when both are at different temperatures [8].

$$
q^{\prime \prime}=-h\left(T_{s}-T_{\infty}\right)
$$

Where;

$\mathrm{h}$ is the convective heat transfer coefficient $\left(\mathrm{W} / \mathrm{m}^{2}{ }^{\circ} \mathrm{K}\right)$

$T_{s}$ is the surface temperature $\left({ }^{\circ} \mathrm{K}\right)$

$T_{\infty}$ is the fluid temperature $\left({ }^{\circ} \mathrm{K}\right)$

3) Radiation: is the energy emitted by a matter that is at non zero temperature. The energy emmitted by radiation field is transported by electromagnetic waves [6].

$$
q^{\prime \prime}=J-G
$$

Where;

$$
\mathrm{J} \text { is the radiosity }\left(\mathrm{W} / \mathrm{m}^{2}\right)
$$

$\mathrm{G}$ is the irradiation $\left(\mathrm{W} / \mathrm{m}^{2}\right)$

$$
J=E+\rho G
$$

$\mathrm{E}$ is the emissive power $\left(\mathrm{W} / \mathrm{m}^{2}\right)$

$\rho$ is the reflectivity

\section{4) Energy Balance:}

The energy balance is given by the equation below:

$$
\frac{\partial}{\partial t}\left(\rho C_{p} T\right)=\frac{\partial}{\partial x}\left(k \frac{\partial T}{\partial x}\right)+\frac{\partial}{\partial y}\left(k \frac{\partial T}{\partial y}\right)+\frac{\partial}{\partial z}\left(k \frac{\partial T}{\partial z}\right)
$$

\section{Fluent Modeling}

This case is modeled using Ansys fluent simulation tool. The solution is updated every 0.5 second with 40 iterations per time step for a total flow of approximately 15 mins. 
In this study, a heat sink is placed in a fluid domain with one inlet and one outlet. The heat sink will be studied under three different heat fluxes 1250, 2500, and $5000\left(\mathrm{~W} / \mathrm{m}^{2}\right)$ at $26^{\circ} \mathrm{C}$ as an ambient temperature.

Because our study is a free convection study, pressure inlet is specified as boundary condition for inlet with a pressure gauge equal to zero. Due to symmetry, half of the domain can be studied as shown in Figure 3.

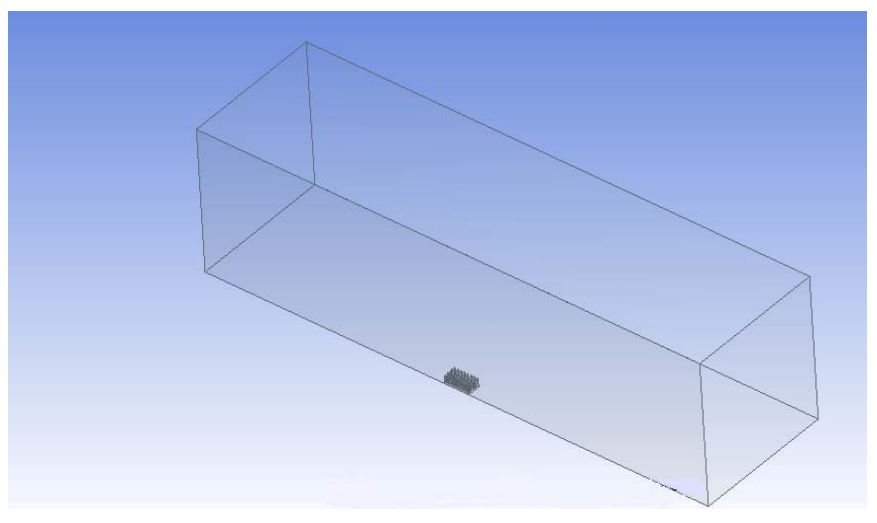

Fig.3. The heat sink and the domain

The domain is divided into 2750000 hexahedral elements as shown in Fig. 4.

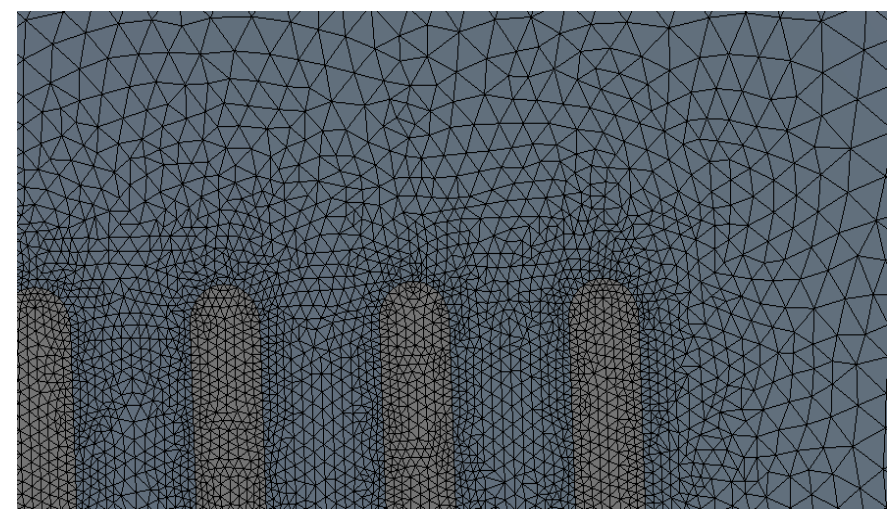

Fig.4. Part of the mesh

\section{RESULTS}

As shown in figure 5, the temperature starts to increase from the ambient temperature $\left(300{ }^{\circ} \mathrm{K}\right)$ to the steady state temperature $\left(326.5^{\circ} \mathrm{K}\right)$. At the beginning, the temperature increases significantly, then its rate decreases with time, that's because, when the surface temperature of heat sink increases, the temperature difference with ambient $\left(T_{s}-T_{\infty}\right)$ increases so that the heat rejected by convection and radiation increases with time until reaching steady state and becomes closer to heat generated by the chip $\left(1250 \mathrm{~W} / \mathrm{m}^{2}\right)$. Eventually, heat generated is equal to heat rejected.

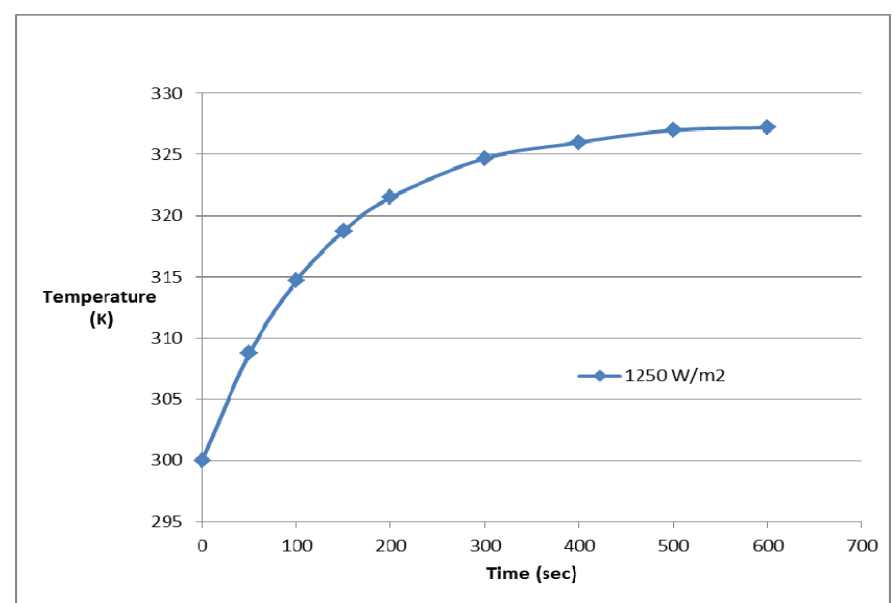

Fig.5. Variation of temperature of heat sink walls with function of time under $1250\left(\mathrm{~W} / \mathrm{m}^{2}\right)$.

The time needed for the heat sink to reach steady state temperature is $9.5 \mathrm{~min}$.

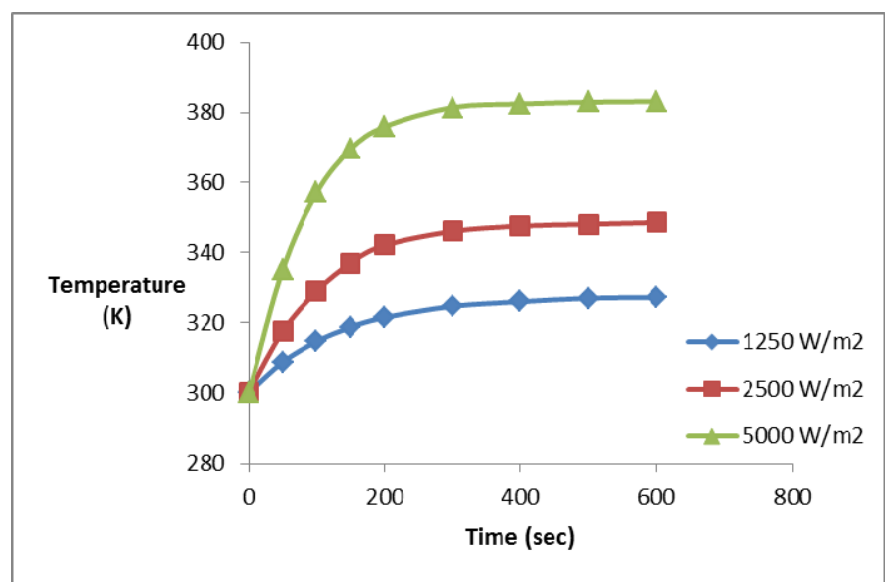

Fig.6. Variation of temperature of heat sink walls with function of time under 1250. 2500 and $5000\left(\mathrm{~W} / \mathrm{m}^{2}\right)$.

As shown in figure 6, it is obvious that when the heat flux increases, the steady state temperature increases, until it reaches 348 and $383{ }^{\circ} \mathrm{K}$ for 2500 and $5000 \mathrm{~W} / \mathrm{m}^{2}$, respectively.

Note that the temperature reached with $5000 \mathrm{~W} / \mathrm{m}^{2}$ is close to the harmful maximum allowable temperature (393 $\left.{ }^{\circ} \mathrm{K}\right)$, so the electronic ship fails to work properly, that's why an additional cooling technique is required. 


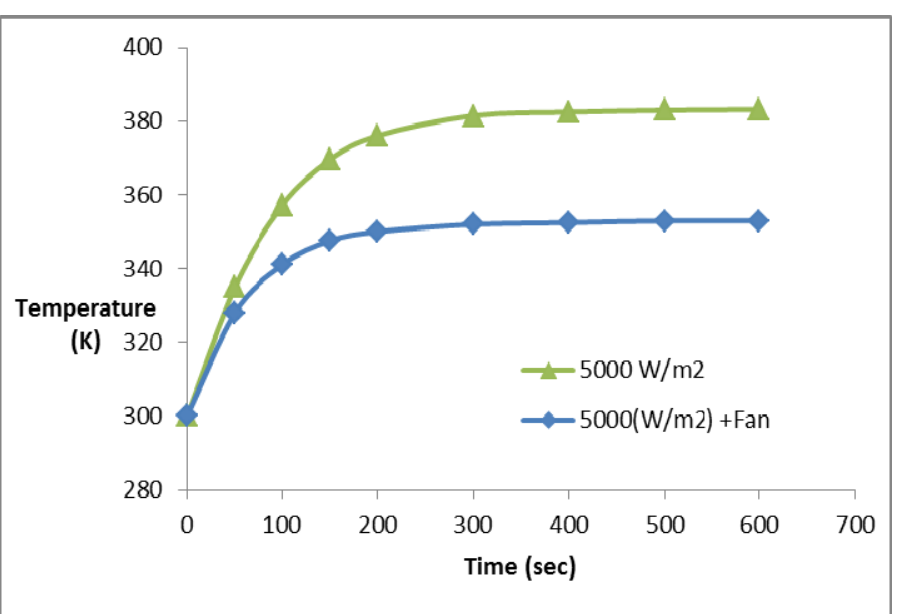

Fig.7. Variation of temperature of heat sink walls with function of time under $5000\left(\mathrm{~W} / \mathrm{m}^{2}\right)$ and forced ventilated.

Figure 7 shows clearly that the forced ventilation decreases the temperature of the heat sink to $353{ }^{\circ} \mathrm{K}$ instead of $383{ }^{\circ} \mathrm{K}$ which is considered an acceptable temperature for a standard operation of electronic chips.

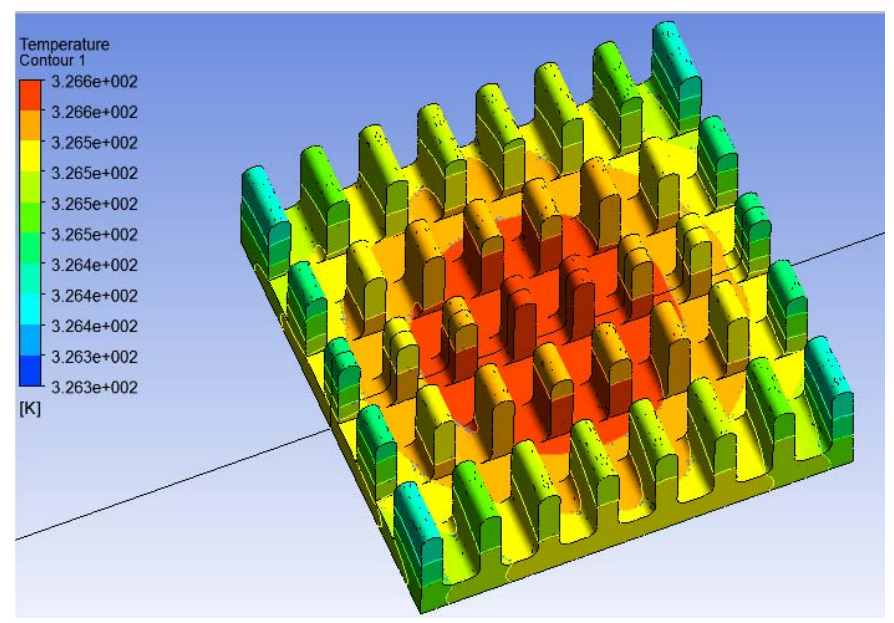

Fig.8. Temperature distribution through the heat sink

Figure 8 clearly shows that the temperature reaches its maximum value at the center of the heat sink, and it decreases gradually when moving away from the center and up to the top of the fins.

From the simulation results shown above, one can conclude that the heat sink might be used as good cooling technique of most of electronic devices. However, traditional heat sinks have some limitations. Based on the later observation, the authors of this paper are currently testing a complementary cooling technique which consists of adding Phase Change Materials (PCMs). The PCM is to be integrated within the heat sink [7].

\section{CONCLUSION}

This paper aims to simulate the heat distribution over a heat sink using Ansys fluent simulation tool to serve the goal of investigating and studying the effect of integrating phase change materials later on into the heat sink in order to improve the cooling performance and consequently increase the efficiency of electronic chips.

\section{REFERENCES}

[1] Hill, M. (n.d.). Cooling of Electronic Equipments"chapter 15". Higher education.

[2] Coskun, A. K. 2010. "Energy-efficient variable-flow liquid coolingg in 3D stacjed architectures." DATE,pp 111-116.

[3] H. Xie, A. Ali. 2011. "The use of heat pipes in personal computers." ITHERM 331-340.

[4] Lasance, Clemens J.M. 2005. "Advanced in high-performance cooling for electronics." Electric cooling magazine 2-8.

[5] Lee, S. (n.d.). How To Select a Heat Sink. Advanced Thermal Engineering.

[6] Kandasamy, Ravi. 2007. "Transient cooling of electronics using phase change material (PCM)-based heat sinks." Elsevier, December 23: 11.

[7] Ahmad hasan, Hassan Hijase, shaimaa Abdelbaqi, Ali Assi, Mohammad Hamdan. "Comparative Effectiveness of Different Phase Change Materials to Improve Cooling Performance of Heat sinks for Electronic Devices." Applied Sciences, 2016: 2-3.

[8] Frank P. Incropera David P. Dewitt, Theodore L. Bergman, Adrienne s. Lavine Fundemantals of Heat and Mass Transfer [Book]. - [s.1.] : John willey and sons, 2006 